\title{
Diskussion zum Referat von Hans R. Brunner
}

\section{Diskussionsteilnehmer:}

HB Dr. HANS R. BRUNNER, Geograph und lic. rer. soc., Zürich (Referent)

KI KLAUS I. ITTEN, Geograph, Zürich (Diskussionsleiter)

PB PETER BUUNZLI, Geographiestudent, Zürich

PH Dr. PAUL HOYNINGEN, Physiker und Wissenschaftstheoretiker, Zürich

ER ERICH RENNER, Geograph, Zürich

CS CHRISTIAN SCHMID, Geographiestudent, Zürich

BW BRUNO WALDER, Geographiestudent, Zürich

PW PIERRE WALTHER, Geograph, Zürich

HW HERBERT WANNER, Geograph, Zürich

AW ANDI WERDER, Geographiestudent, Zürich

KI: Die Situation damals scheint mir am Anfang wichtig zu sein, weil wir ja nicht dabei waren, als diese Dinge in Zürich geschahen; als die zentralen Figuren der Landschaftsschule ihre Ideen entwickelt haben. Studenten und Dozenten lebten damals an der Freiestraße sehr nahe beisammen. Beim gemeinsamen Mittagsmahl haben Dozenten und Assistenten diskutiert. HANS CAROL war Oberassistent unter Professor BOESCH. Könnten Sie sich zur Atmosphäre damals noch etwas äußern?

HB: In Zürich gab es damals wie heute zwei Geographische Institute, eines an der ETH und eines an der Universität. Die ETH hatte immer weniger Studenten als die Universität. An der ETH konnte man seit 1925 das Diplom in Geographie machen. Der erste Abschluß kam erst nach dem 2. Weltkrieg zustande. Der damalige Chef des Geographischen Institutes der ETH war HEINRICH GUTERSOHN, Jahrgang 1899. Er lebt heute noch hochbetagt. Sein Name hat vielleicht noch gefehlt hier. GUTERSOHN ist bekannt geworden durch seine fünfbändige Geographie der Schweiz, ein Standardwerk. Darin versuchte er das, was sein langjähriger Assistent WINKLER methodologisch begründet hatte. GUTERSOHN hat versucht, dies in einer ungeheuren Arbeitsleistung in die Praxis umzusetzen. Er hat sämtliche Landschaften der Schweiz nach möglichst objektiven Kriterien beschrieben. Ich kann Ihnen nur empfehlen, das Buch einmal hervorzunehmen. Greifen Sie diejenigen Landschaften heraus, die Sie durch eigene Erfahrung kennen; dann werden Sie jedoch erkennen, wie substanziell die Beschreibungen sind. Zur Situation ist noch zu sagen, daß schon immer eine gewisse Spannung zwischen den beiden Instituten herrschte. Vor allem bei den methodologischen Diskussionen spielten diese Spannungen immer wieder mit.

Eine sehr wichtige Stellung hatte ERNST WINKLER dadurch, daß er während vieler Jahre Redaktor der Geographica Helvetica war. Hier hatte er die Möglichkeit, seine ureigensten Gedanken auszubreiten. Er konnte vor allem auch selektionieren. $1960 \mathrm{kam}$ einer der bedeutendsten Artikel über die Landschaft von SZAVA-KOVATS heraus. Ein ungarischer Geograph und Philosoph, der sich stark mit diesen Dingen beschäftigt hat. Er kam zu dem Fazit, daß Landschaft eine Fiktion sei. ERNST WINKLER zögerte lange. SZAVA-KOVATS wollte dann noch einen zweiten Artikel publizieren. Dies hatte wieder einen langen Schriftwechsel zwischen ihm und wINKLER zur Folge, bis die Verantwortung auf den neuen GHRedaktor HANS BOESCH überging. Dieser publizierte dann den Artikel in den 70er Jahren.

PH: Ich habe eine Frage, die mir in einem anderen Zusammenhang über den Weg gelaufen ist. Wie ernst ist bei FRIEDRICH RATZEL der geographische Determinismus gemeint? Heißt das, daß die geographischen Einheiten, also die Lithosphäre und die Atmosphäre usw., daß diese die Dinge, die in den sozialen Bereichen passieren, determinieren? Wie weit geht diese Determination?

HB: Wenn man RATZELs Schriften liest, hat man eine Schwierigkeit: Manchmal hat man das Gefühl, er sei Determinist, manchmal scheint er auch Probabilist zu sein. Er nimmt auch Dinge zurück, die er einige Seiten vorher behauptet hat. Das Ganze ist vielfach noch in eine literarische Form gebracht, wodurch alles diffus wird. Es gibt Leute, die haben ihm vorgeworfen, ein sehr strenger Determinist zu sein, und sehr oft wird er in die Nähe des Nationalsozialismus gerückt, deren Wegbereiter er für einige gewesen sein soll.

PW: Ich möchte das Thema Landschaftsbeschreibung zur Sprache bringen. Du hast das Buch von HEINRICH GUTERSOHN als trivial bezeichnet. Tatsache ist, daß es immer noch das beste Buch ist, wenn man sich in ein Gebiet unverbindlich einlesen will. Meine Frage ist nun, ob die Domäne Landschaftsbeschreibung in der Geographie zu wenig ernst genommen wird oder ob dieses Gebiet einfach trivial ist?

HB: An sich ist die Landschaftsbeschreibung sicher nicht trivial. Man darf nur nicht davon ausgehen, man müsse alles möglichst gleichmäßig und vollständig beschreiben. Die Beschreibungen beginnen ja immer mit der Größe der Landschaft, dann kommt das bekannte länderkundliche Schema hinein. Ich frage mich einfach: Wozu dieser riesige Aufwand? Wem nützt das? Ist es unbedingt notwendig eine fünfbändige Geographie der Schweiz zu schreiben, wenn man einen mehr oder weniger flüchtigen Eindruck über die Grundvorgänge der Schweiz erhalten will? Die Probleme werden in diesen Beschreibungen meist ausgeklammert. Dies ist ja auch der Vorwurf, welcher der Landschaftsgeographie gemacht wird, also daß diese Art von Geographie zu wenig 
problemorientiert sei und an den Problemen vorbeisehe. Man kann auch problemorientierte Landschaftsbeschreibungen machen, dann ist es wieder eine andere Sache.

PB: Kann man sagen, daß diese Landschaftsgeographie ein Paradigma sei? Ist sie überhaupt eine Wissenschaft?

HB: Dies kommt darauf an, was Du unter Paradigma verstehst?

PB: So in etwa dies, was KUHN und LAKATOS darunter verstehen.

HB: Mit einigem Wohlwollen kann man die Landschaftsgeographie als Paradigma betrachten. Es ist eine Anleitung, ein Standard gegeben, nämlich die Descriptio, die Beschreibung.

PB: Aber BARTELS beispielsweise bezeichnet die Landschaftsschule als eine Richtung mit Leerformelcharakter. Es sei zwar füllemäßig etwas vorhanden, aber inhaltlich überhaupt nichts.

HB: Dies hat mit dem Paradigma nichts zu tun. Trotz des Leerformelcharakters kann die Landschaftsgeographie ein Paradigma sein, nämlich ein Paradigma von Tautologien.

PB: Aber wenn man dieses Paradigma nur durchsetzen kann, indem man Gegner wie einen EISEL nicht hat, dann ist das gar keine Wissenschaft, weil gar kein Diskurs vorhanden ist, sondern nur ein monopolisierter Diskurs.

HB: Dies gibt's natürlich überall. Es gibt auch verschiedene Definitionen von Wissenschaft. Ich kann Wissenschaft soziologisch definieren. Dies ist etwas anderes, als wenn ich Wissenschaft erkenntnislogisch zu definieren versuche.

ER: Ich möchte noch eine Bemerkung machen zur «Geographie der Schweiz» von HEINRICH GUTERsOHN. Wenn eine Tätigkeit den Anspruch erhebt, Wissenschaft zu sein, dann muß sie nachvollziehbar sein. Wenn jedoch der Anspruch einfach darin besteht, ein Gebiet beschreiben zu wollen, um eine Einführung zu geben, dann ist die Frage, ob, um der Nachvollziehbarkeit willen, immer wieder alles nach dem länderkundlichen Schema abgehandelt werden soll.

HB: Ich finde es schade, daß wertvolle Zeit eingesetzt wurde, um ein solches Werk zu schreiben, welches, kaum erschienen, im Grunde genommen bereits wieder veraltet ist. Es wurde in den späten fünfziger Jahren geschrieben. Die ganzen Probleme, welche danach kamen und dies sind eigentlich diejenigen Probleme, welche uns heute beschäftigen sollten, diese fanden im Werk keinen Niederschlag. Dann wurde noch eine zweite Auflage herausgegeben, ohne das Werk zu überarbeiten. Dies finde ich sehr fragwürdig.
PW: Ich möchte behaupten, daß beispielweise das Buch von EISEL einen kleineren Benützerkreis hat als das Buch von Gutersohn, welches auch heute noch die wesentlichen Probleme aufzuwerfen vermag. Wenn man beispielsweise eine Einführung in ein $\mathrm{Ge}$ birgstal braucht, liefert GUTERSOHN eine brauchbare Darstellung.

HB: Ich möchte dies schon etwas relativieren. Leider gibt es keine Untersuchungen über den Benützerkreis solcher Bücher. Aber in der Schweiz gibt es kleinere Werke, die einen ebensoguten Überblick vermitteln. Beispielsweise die Schrift von EMIL EGLI über die Geographie der Schweiz finde ich besser als das fünfbändige Werk von GUTERSOHN. Wer liest schon fünfbändige Werke. Ich lese lieber das prägnantere und stilistisch ansprechendere Buch von EGLI, das auch ins Englische übersetzt wurde.

KI: Dabei dürfen wir das Ausbildungsprofil nicht vergessen. Damals wie heute studierte man Geographie vor allem um Mittelschullehrer zu werden. Sehr viele der genannten Geographiewerke sind davon geprägt und sind darauf ausgerichtet, daß man sie in der Schule verwenden kann. Dort ist das starre Schema sicher gut brauchbar. Heute haben wir natürlich neben der Ausbildung zum Mittelschullehrer auch andere Ansprüche.

HB: Die ganze Landschaftsdiskussion muß auch unter diesem Aspekt betrachtet werden. Wozu studierte man überhaupt Geographie? Bis vor wenigen Jahren praktisch ausschließlich für das Höhere Lehramt. Hier hat sich ein enormer Wandel abgespielt. In der Lehre hat sich in diesem Zusammenhang viel zu wenig abgespielt. Wenn man heute gegen das Landschaftskonzept ist, so hängt das auch damit zusammen, daß wir ganz andere Anforderungen an das Fach Geographie stellen müssen als noch vor 15 bis 20 Jahren. Die Anpassungen gehen vor allem an den schweizerischen Hochschulen viel zu wenig weit.

ER: Die Landschaftsgeographie ist eben auch für die Schule nicht geeignet. Stellen sie sich die armen Schüler vor, die immer wieder mit demselben Schema gelangweilt werden.

$\mathrm{KI}$ : Ein Grund, wieso BOESCH das Geosphärenmodell von CAROL in der Lehre immer noch durchgesetzt hat, ist, daß es im Gegensatz zu vielen diffusen Begriffen etwas Haltbares darstellt. Auch ist es sehr einfach zu erklären.

HB: Man sollte einmal überprüfen, ob das Modell von CAROL nicht eine Neuauflage des sehr viel älteren länderkundlichen Schemas ist, welches auf Hettner zurückgeht.

HW: Ich möchte am Modell von CAROL folgendes kritisieren: Wenn es in der Schule verwendet wird, dann meistens nicht als solches, sondern die fünf Sphären werden als Inhaltsverzeichnis für eine Län- 
derkunde verwendet, indem man zuerst über die Geologie, dann über das Klima, über die Vegetation, über die Bevölkerung und über die Wirtschaft spricht. Die Beziehungen der verschiedenen Sphären untereinander werden so gar nicht eingelöst.

HB: Hinter der Landschaftsidee steht die Ganzheitsideologie, die Gesamtsynthese. Zu dieser ganzheitlichen Synthese kommt es meistens eben nicht. Man glaubt durch das Aneinanderreihen, durch das Auflisten der verschiedenen Sphären, die Synthese schon geleistet zu haben. Aber damit würde die eigentliche Arbeit des Landschaftsgeographen erst beginnen. Aber dieses Problem wurde meistens nicht gelöst.

HW: Deshalb verstehe ich auch nicht, wieso Du sagst, daß das Buch "Geographie der Schweiz» von GUTERSOHN die Anwendung der methodologischen Grundzüge wINKLERs sei. Da sehe ich eine gewisse Diskrepanz zwischen der Methodologie WINKLERs und dem Werk von GUTERSOHN, welches viel mehr einer Faktensammlung, einer Superstatistik entspricht als einer Synthese.

HB: Du mußt einmal das Schlußwort von GUTERSOHN lesen. Darin hat er eigentlich zugegeben, daß sein Werk ein Torso sei. Er schreibt dort, daß man jetzt eigentlich noch die Synthese machen müßte. Aber er wolle dies jüngeren Kräften überlassen. In den letzten Jahren gab es an unserem Institut Bestrebungen, auf den achtzigsten Geburtstag von HEINRICH GUTERSOHN (1979) diesen zweiten Teil, also diese Gesamtsynthese, in Angriff zu nehmen. Die ganze Sache ist dann hoffnungslos stecken geblieben. Erstens ist die Idee etwas aus der Mode gekommen und zweitens kann man sich einfach nicht vorstellen, wie eine solche Synthese zu bewerkstelligen ist. Man hat etwa zwanzig Leute eingeladen, und jeder sollte irgend einen Teil dazu beitragen. Da eine Gesamtkonzeption nicht vorhanden war, wäre es im Grunde genommen wieder darauf hinausgekommen, daß jeder irgend etwas geschrieben hätte und daß das Ganze dann so mosaikartig zusammengestellt worden wäre. Aber das hängt nicht einmal so sehr am Konzept selber, sondern es ist ein Personalproblem. Keiner traute dem anderen zu, in der Lage zu sein, diese Synthese machen zu können. Der einzige, welcher in Frage kam, war WINKLER.

$\mathrm{PH}$ : Es verwirrt mich immer, wenn von der Geographie als Synthesewissenschaft gesprochen wird und das dann so verstanden wird, wie wenn die Gesamtsynthese über die fünf Sphären in der Schweiz auf einen Schlag geleistet werden müßte. Das wäre dann der Abschluß der Geographie der Schweiz. Anschließend müßten alle Geographen entlassen werden. Ich verstehe es deswegen nicht, weil das kein sinnvoller Anspruch sein kann. Sondern der Anspruch kann doch nur sein, einen Teil der Korrelatio- nen in einem Forschungsprojekt zu untersuchen, und nicht die Geographie in einem Streich mit weiteren fünf Bänden abzuschließen.

HB: Diese Forderung wurde dadurch abgefangen, $\mathrm{da} ß$ man die Zeitdimension in die Geographie einführte. Die Zeitdimension in der Geographie war lange umstritten. Das wäre dann einfach eine Synthese über den gegenwärtigen Stand und weil ja die Kulturlandschaftsentwicklung weitergeht, so hätten auch künftige Geographengenerationen noch genügend zu tun gehabt, um neue Synthesen zu fabrizieren.

PH: Aber das wäre analog der Forderung, daß die Molekularbiologen sämtliche Aspekte jetzt vorhandenen Lebens vollständig zusammenschreiben sollten und die Zukünftigen nur noch die industrieinduzierten Mutationen zu beschreiben hätten. Das ist doch eine Forderung, die überrissen ist. Damit kann man die eigene Wissenschaft schon kaputtmachen. Daran verzweifelt man, das ist klar. Es kann doch immer nur partielle Resultate geben.

HB: Aber solche Forderungen wurden aufgestellt und zwar eben, indem man sagte, man müsse die Landschaft ganzheitlich beschreiben, d. h. man muß alles beschreiben, was $d a$ ist und dies möglichst objektiv.

PH: Dem stimme ich ja zu als Programm. Aber man darf doch nicht die vollständige Realisierung des Programms als einzelnes Forschungsprojekt betrachten. Das klafft auseinander. Die Molekularbiologen wollen in der Tat das Leben als Ganzes verstehen, aber sie schauen $z$. B. wie eine Zellwand auf ein Chemikal reagiert. Deshalb bleibt doch als Leitidee das Verständnis des Lebens da. Auch wenn man das in den nächsten $100 \mathrm{Jahren}$ nicht vollständig machen kann. Mir scheint, daß diese beiden Ebenen in der Diskussion und auch in dem, was ich bisher gelesen habe, immer wieder durcheinander gehen: die sinnvolle Eingrenzung eines Forschungsprojekts im Unterschied zu dem globalen Ziel, das man mit einer Wissenschaft anstrebt.

$\mathrm{KI}$ : Es war ein entscheidender Schritt, daß von einer statischen Geographie weggegangen wurde zu einer dynamischen Geographie. Damit verließ man auch das Landschaftskonzept, welches etwas mit der statischen Geographie verwurzelt ist und strebte eine gesellschaftsbezogene Geographie an. Dieses Feld war dann so offen, daß man sagte: Geographie ist, was Geographen tun.

AW: Meine Frage zielt auf einen anderen Problemkomplex. Können Sie die Auswirkungen der jüngeren Landschaftsschule auf andere Theorien skizzieren wie zum Beispiel auf den regionalwissenschaftlichen Ansatz, wo ja diese Synthese nicht explizit angestrebt wird. Gibt es überhaupt Bezüge 
vom Geosphärenmodell zum regionalwissenschaftlichen Ansatz, welcher vor allem im angelsächsischen Bereich theoretisch und methodisch verfeinert wurde?

HB: Die Regionalforschung ist, wie Sie selbst gesagt haben, eine angelsächsische Angelegenheit, welche aus den USA kam, und sich im Anschluß an die jüngere Landschaftsschule bei uns auszubreiten begann, wie auch die ganze quantitative Geographie. Aus einem Unbehagen am bisherigen Betrieb, d.h. an der traditionellen Geographie, welche man seit etwa 100 Jahren betrieb - auch wegen der Diskrepanz zwischen Anspruch und Wirklichkeit in der Landschaftsgeographie -, hat man sich um so eher auf diese neuen Ansätze gestürzt, welche an sich nichts zu tun haben mit dem Landschaftskonzept. Entsprechend stark hat man sich dann mit quantitativen Techniken und mit dem Computer befaßt. Als eine Reaktion auf das Ungenügen der Landschaftsschule hat man ganz andere Strömungen meist kritiklos übernommen.

AW: Aber steckt in der Diskussion über den Regionsbegriff nicht eine Wiederholung der Diskussion über den Landschaftsbegriff?

HB: Das glaube ich nicht. Wobei eine gewisse innere Verwandtschaft zwischen dem CAROL'schen Landschaftsbegriff und dem Begriff der Region besteht. Die Region ist einfach eine rein pragmatische Abgrenzung des Untersuchungsgebietes. Darauf läuft es meines Erachtens auch bei CAROL hinaus, wenn man ihn daraufhin liest. Mehr ist es kaum. Dies ist, was H.D.SCHULTZ als die Selbstauflösung der Landschaftsgeographie bezeichnet. Im Grunde wird durch die Konzeption der jüngeren Landschaftsschule, wie etwa bei CAROL, der Landschaftsbegriff überflüssig. Damit erübrigt sich auch die Diskussion. Nach CAROL kann man die Landschaft nach $\mathrm{x}$-beliebigen Kriterien und Interessen abgrenzen. Die Abgrenzung ist kein Problem mehr. Damit fällt die Diskussion um den Landschaftsbegriff dahin, da sie über weite Strecken hinweg eine Diskussion um die Abgrenzung war. Wenn man nun eine Landschaft abgrenzen kann, wie man will, ist die Diskussion fertig.

CS: Könnten Sie noch etwas sagen über die Bedeutung der Landschaftsgeographie heute? Meines Wissens werden immer noch Forschungsarbeiten mit diesem Ansatz gemacht.

HB: Zum Teil ist dies ein Generationenproblem. Die älteren Geographen und ihre Schüler (sofern die Lehrer überzeugend auf ihre Schüler einwirken konnten) haben dieses Konzept noch beibehalten. Es wird auch weiterhin Leute geben, die daran festhalten. Es ist auch durchaus möglich, daß die ganze Diskussion wieder von vorne beginnt. Gewisse An- zeichen dafür sind vorhanden; nicht zuletzt auch dieses Seminar zeigt doch, daß ein gewisses Interesse an solchen Fragen besteht. Ähnlich ist es auch mit der Diskussion um die Länderkunde, das zweite heiße Eisen in der Geographie. In gewissem Sinne ist diese Diskussion verwandt mit der Landschaftsdiskussion. 1969 glaubte man, daß dieses Thema ein für alle Male aus den Traktanden verschwinden würde. Aber in den letzten Jahren hat man gesehen, daß die Länderkunde wieder vermehrt aufkommt, vor allem auch die Diskussion um die Länderkunde. Dies hängt natürlich auch mit personellen Strukturen zusammen. Einer, welcher die Länderkunde in einem modernisierten Sinne verteidigt, ist EUGEN WIRTH aus Erlangen, welcher zufälligerweise - oder eben auch nicht zufälligerweise - momentan Vorsitzender der Deutschen Geographen ist.

HW: Du hast nun das Konzept der Landschaftsgeographie daran kritisiert, daß die versprochene Synthese nirgends habe eingelöst werden können. Ich möchte nun aber ein Beispiel zur Diskussion stellen, in welchem eine solche Synthese angestrebt wurde: HANS BOESCH, welcher die Begriffe Geosphäre und Geomer von CAROL propagierte, hat u. a. zwei Länderkunden geschrieben, über die USA und über Japan. Darin hat er die Probleme aus verschiedenen Sphären zueinander in Beziehung gesetzt. Diese Werke umfassen auch nicht fünf Bände, sondern ca. 300 Seiten. Ich frage Dich, wie Du diesen Versuch von BOESCH, mit Hilfe des Geosphärenkonzeptes eine Synthese zu bewerkstelligen, beurteilst?

HB: Dazu muß ich sagen, daß ich mir diese beiden Bücher von BOESCH auch ohne das Geosphärenmodell vorstellen kann. Das Geosphärenmodell ist für die Boeschbücher nicht so fundamental. Da kommen ganz andere Fähigkeiten von HANS BOESCH zum Tragen.

KI: Aber ich glaube doch, daß HANS BOESCH aus seiner Haut nicht raus konnte. Wenn er das Geosphärenmodell in den Büchern auch nicht expliziert, so finden wir die Strukturen doch immer wieder. Dieses Modell hat ihn zeitlebens beschäftigt, wie auch die Abgrenzung einer Landschaft. Er konnte dieses Thema nicht mehr zu Ende bringen. Daher finden wir jetzt auch das Werk über «die Grenzen», welches von Frau KISHIMOTO redigiert worden ist.

PH: Ich verstehe überhaupt nicht, wie man den Modellbegriff mit der Vorstellung der Geosphäre zusammenbringen kann. Denn wofür soll denn die Geosphäre ein Modell sein?

KI: Es ist nicht so einfach, die Sphären voneinander abzugrenzen.

PH: Das ist eine andere Frage, wie gut ich diese Unterscheidung machen kann. Aber gerade das leistet doch kein Modell. Ich spreche doch von einem Mo- 
dell, wenn etwas für etwas anderes steht. Wenn ich z. B. ein Modell eines Architekten nehme, dann ist doch das Modellhaus des Architekten nicht das reale Haus.

HW: Ich stelle mir das so vor, daß das Geosphärenmodell ein Weltmodell ist. Unter Modell verstehe ich eine Konstruktion, mit deren Hilfe man gewisse Probleme diskutieren kann. Die Physiker beschreiben die Ereignisse auf einem Billardtisch mit dem Strahlensatz und dem Satz des vollelastischen Stoßes. Diese Sätze können als dem Billardtisch hinreichend ähnlich bezeichnet werden, aber nur, um den Lauf der Kugeln zu beschreiben. Analog ist für mich das Geosphärenmodell hinreichend ähnlich der realen Welt, aber nur um Korrelationen zwischen den Sphären zu beschreiben.

PH: Ich würde das nicht als Modell, sondern als eine Idealisierung bezeichnen. Eine andere Frage ist die Abgrenzung der Sphären. Wenn ich ein Haus anschaue, das aus Stein gemacht ist: gehört dann dieses Haus zur Lithosphäre oder zur Anthroposphäre? Das ist schon schwierig zu sagen. Das Haus ist ein Artefakt, aber aus Naturstoff. Da wird's schwierig, genau abzugrenzen. Das ist aber eine Frage der Schärfe der Abgrenzungen. Die Abgrenzungen, wie sie vorgestellt wurden, sind Idealisierungen. Aber das ist etwas anderes als ein Modell. Jezt nehme ich noch ein Beispiel aus der Physik. Beim Tröpfchenmodell aus der Kernphysik weiß ich genau, daß der Atomkern kein Tröpfchen ist, sondern aus Protonen und Neutronen zusammengesetzt ist. Aber ich behandle ihn als homogenes Flüssigkeitströpfchen. Ich setzte also etwas anderes ein, um damit arbeiten zu können. Ein anderer Fall ist eine etwas idealisierte Beschreibung, aber ich würde das nicht ein Modell nennen.

KI: Wenn wir von BOESCH sprechen, möchte ich die Gelegenheit benützen, um einen seiner Verdienste zu erwähnen. Du hast gesagt, daß HANS BOESCH die Entpolitisierung der deutschen Geographie nach 1945 gefördert hat. Er hat der Geographie geholfen, aus ihrem Paradigma herauszukommen, indem er deutsche Geographen nach Zürich geholt hat. Damit soll er der deutschen Geographie zu neuem Ansehen verholfen haben.

HB: Vor allem jenen deutschen Geographen, die offenbar oder angeblich nicht mit der Geopolitik in Berührung gekommen waren. Das ist z. B. CARL TROLL, der sich auf die rein naturwissenschaftliche Geographie zurückgezogen hatte.

PW: Ich glaube, daß das Geosphärenmodell außerordentlich lange erhalten geblieben ist, weil in den 60 er Jahren die Diskussion um die Landschaftsgliederung und um die Probleme der naturräumlichen Gliederung gerade im deutschen Sprachbereich eine große Rolle gespielt haben. Diese Forschungsgegen- stände sind heute zum Glück etwas aus dem Blickfeld geraten. Aber auch heute werden noch solche Arbeiten gemacht. Beispielsweise die Arbeit von Schilter "Versuch einer Landschaftsgliederung und -Typologie der Schweiz». Diese Arbeit wurde 1975 noch unter Ernst Winkler gemacht. Der Arbeit liegt das Geosphärenmodell zugrunde. Zuerst wurden analytische Karten gezeichnet: Gewässernetz, Vegetation usw. Diese wurden dann übereinander gehalten, um daraus eine Landschaftstypologie zu kreieren. Ich hoffe, daß man heute von solchen Ansätzen weggekommen ist.

HB: Vor allem weil dies eine falsche Objektivität vermittelt. Man glaubte durch das Übereinanderkopieren von verschiedenen Dingen und ihren Grenzen eine optimale und objektive Abgrenzung zu erhalten, welche die Landschaft dann definierbar macht. Damit hätte man das Objekt eingekreist und für alle Zeiten festgenagelt. Das ist im Grunde genommen ein falscher Anspruch.

BW: Inwiefern unterscheidet sich wissenschaftstheoretisch die Anwendung der quantitativen Geographie vom Schema der Länderkunde? Ich denke an Arbeiten, in welchen man versucht hat, zwischen verschiedenen Sphären Bezüge herzustellen und die Korrelationen zu berechnen, um daraus induktiv ein Modell zu erstellen.

HB: Da steckt ein ganz anderer Anspruch dahinter. In der Länderkunde geht es auch um eine ganzheitliche Wiedergabe der Erscheinungen eines Landes. In der quantitativen Geographie kann man damit kaum etwas anfangen. Hier geht es um Variablen, die man miteinander vergleicht. Man versucht, zwischen verschiedenen Meßreihen Korrelationen zu finden. Der Ganzheitsanspruch fällt bei der quantitativen Geographie von vornherein weg.

BW: Ich glaube, daß er eben dort nicht wegfällt, wo Fakten einfach wahllos gesammelt und wo beschreibende Variablen angehäuft werden, um aus dem $\mathrm{Da}$ tenhaufen durch Korrelationsberechnungen zu einem Modell zu gelangen.

HB: Aber für die Modellbildung in der quantitativen Geographie wählt man ja gewisse Variablen aus. Es wird also eine Selektion der Variablen gemacht, was in der Landschaftsgeographie nicht der Fall ist, hier muß alles hineinkommen, wenigstens nach den strengen Methodologen.

AW: Aber man kann ja noch einen Schritt weitergehen als nur Korrelationsrechnungen zu machen, z. B. indem man Simulationen rechnet. Dort sehe ich eine gewisse Renaissance des Ganzheitsbegriffes, indem mit numerischen Verfahren riesige Differenzialgleichungssysteme aufgelöst werden können, die dann schlußendlich eine Kurve über das Systemverhalten produzieren. Das ist dann die operationalisierte Synthese. 
KI: Dort verlieren sie aber die Gesellschaftsrelevanz, indem sie ein Problem als Kurve darstellen, verlieren sie den Bezug zu den Leuten, welchen Sie das Problem darlegen wollen.

HB: Ob das eine Synthese im Sinne der alten Landschaftsgeographie ist, weiß ich nicht.

PW: Das Geosphärenmodell hatte auch dort Nachwirkungen, wo es auf Naturlandschaften angewendet wurde. Die landschaftsökologischen Modelle sind Abkömmlinge des Geosphärenmodells, z. B.im MAB, dem UNESCO-Projekt «Man and Biosphere», werden solche Modelle verwendet.

HB: Aber das Wesentliche am Geosphärenmodell sollte ja die Korrelation zwischen der geistigen und der Natursphäre sein. Wenn nun nur noch die Naturlandschaft betrachtet wird, würde man den Hauptinhalt preisgeben.

\section{Literaturbesprechung}

KOECK Helmuth: Theorie des zielorientierten Geographieunterrichts. Aulis Verlag Köln, 205 S., 17 Abb., 1980, DM 38.--.

Der Autor versucht mit diesem Werk, einem "zielorientierten" Geographieunterricht eine theoretische "Behausung" zu schaffen. In diesem Unterricht richtet sich die Stoffauswahl nach bestimmten vorgegebenen (Lehr- oder Lern-) Zielen. Als Ziel des Unterrichts überhaupt wird die "Befähigung zur rationalen Daseinsgestaltung" festgelegt; der Beitrag des Geographieunterrichts besteht in der Vermittlung von 'Raumverhaltenskompetenz", d.h. der Fähigkeit, das menschliche Dasein "vom Raum und seinen Erscheinungen her... rational zu gestalten". Dieser Unterricht fusst auf einem Curriculum, das sich einerseits an den vorgegebenen Zielsetzungen, andererseits an der modernen geographischen Wissenschaft orientiert. Das Curriculum wird auf der Basis von fünf Daseinsgrundfunktionen (Wohnen, arbeiten, sich versorgen, sich erholen, am Verkehr teilnehmen - als "raumrelevante Lebenssituationen") konstruiert und schliesst ausdrücklich naturgeographische Aspekte nicht aus, wie denn überhaupt der Verfasser eine Spaltung unseres Faches in physische und Anthropogeographie ablehnt.

Zwei abschliessende Kapitel setzen sich theoretisch mit den Lernprozessen, die zum angestrebten Ziel führen, sowie der Messung des Lernerfolges auseinander. Eine Zusammenfassung, die sich mancher Leser wohl wünschen möchte, fehlt, doch ist das Literaturverzeichnis recht umfangreich.

Das vorliegende Werk dürfte für die meisten Geographielehrer nicht einfach zu lesen sein. Als Habilitationsschrift, eingereicht an der Pädagogischen Hochschule Westfalen-Lippe, Abteilung Münster, wendet sich die Publikation wohl primär an den Wissenschafter, nicht an den Praktiker. Sie vermittelt keine didaktischen Tips, sondern stellt ein Theoriegebäude vor für einen Geographieunterricht, dem manche Kollegen (zumindest in der Schweiz) mit einiger Skepsis gegenüberstehen. Doch könnte es ein fruchtbares Unterfangen sein, den eigenen Unterricht einmal wieder auf seine Fundamente hin zu überprüfen. Die kritische Auseinandersetzung mit dem vorliegenden Buch vermöchte hierzu sicher wertvolle Denkanstösse liefern, sei es in Richtung von Neuerungen, sei es aber auch in der Erkenntnis, Bewährtes beizubehalten (und z.B. nicht gerade alles Streben im Schulegeben über den Leist der "rationalen Daseinsgestaltung" zu schlagen!).

Die Lesbarkeit des Werkes wird beeinträchtigt durch häufige lange, verschachtelte Sätze, durch zeilenlange Quellenhinweise im Text (statt in Fussnoten) sowie durch die Manie des Verfassers, einzelne Worte durch Klammern oder Schrägstriche miteinander zu verbinden, z.B.'"be-/ über-/durchgestanden" (p.22), "Schüler- und Lehrer-(Gesellschafts-) interesse/-intention" (p.14).

Was aber dringend Not täte - und hier steht das hier besprochene Buch nicht allein - , wäre, dass sich die Theoretiker der Erziehungswissenschaften einer Sprache bedient, die auch dem einfachen Praktiker Satz für Satz leicht verständlich wäre. Wohl kommt keine Wissenschaft ohne ein gewisses Mass an Fachidiom aus. Doch was taugt es dem Lehrfach Geographie, wenn der Lehrer, einem vermeintlichen Rufer in der wïste folgend, der ihm grünere Gestade verheisst, sich schliesslich im Urwald des theoretischen Fachjargons doch nicht zurechtfindet ?

GH $4 / 81$ Jörg Roth-Kim, Zürich 